\title{
Section $E 6$ of the ACMG technical standards and guidelines: Chromosome studies for acquired abnormalities
}

This updated Section E6 has been incorporated into Section E: Clinical Cytogenetics of the 2005 ACMG Standards and Guidelines for Clinical Genetics Laboratories and supersedes the previous section E6.

Betsy Hirsh, PhD ${ }^{1-3,8}$, Arthur R. Brothman, PhD ${ }^{1,2,4,8}$, Peter B. Jacky, PhD ${ }^{1,2,5,8}$, Kathleen W. Rao, PhD ${ }^{1,2,6,8}$, and Daynna J. Wolff, $P h D^{1,7,8}$

Key Words: Cancer, cytogenetics, chromosomes, chromosome guidelines, cytogenetic analyses, cancer cytogenetics

Disclaimer: These standards and guidelines are designed primarily as an educational resource for clinical laboratory geneticists to help them provide quality clinical laboratory genetic services. Adherence to these standards and guidelines does not necessarily ensure a successful medical outcome. These standards and guidelines should not be considered inclusive of all proper procedures and tests or exclusive of other procedures and tests that are reasonably directed to obtaining the same results. In determining the propriety of any specific procedure or test, the clinical molecular geneticist should apply his or her own professional judgment to the specific clinical circumstances presented by the individual patient or specimen. It may be prudent, however, to document in the laboratory record the rationale for any significant deviation from these standards and guidelines.

E6 Most cytogenetic analyses to detect and characterize acquired chromosomal abnormalities are performed to diagnose or monitor a premalignant or malignant disorder.

\section{GENERAL CONSIDERATIONS}

E6 (a) A patient with an acquired clonal chromosomal abnormality or one who is at high risk for developing such an abnormality may have multiple cytogenetic studies during the course of his or her disease.

E6 (b) Tissue processing, analytical variables and turnaround times should be determined by the laboratory based on the indication for the cytogenetic referral (e.g., initial diagnosis vs. follow-up studies; pre- vs. post-transplant studies) and the clinical application of the cytogenetic results (e.g., selection of therapy).

E6 (c) All results should, to the extent possible, be interpreted in the context of other clinical and laboratory findings.

E6 (d) The laboratory director and staff should be familiar with the various recurrent chromosome abnormalities associ-

From the ${ }^{1}$ ACMG Laboratory Quality Assurance Cytogenetic subcommittee; ${ }^{2}$ ACMG Laboratory Quality Assurance Committee; ${ }^{3}$ University of Minnesota Medical School; ${ }^{4}$ University of Utah School of Medicine; ${ }^{5}$ Kaiser Permanente, NW; ${ }^{6}$ University of North Carolina; ${ }^{7}$ Medical University of South Carolina; ${ }^{8}$ American College of Medical Genetics.

American College of Medical Genetics, 9650 Rockville Pike, Bethesda, MD 20914-3699.

To review Section $E$ in its entirety, please visit the ACMG website at www.acmg.net.

Go to www.geneticsinmedicine.org for a printable copy of this document.

Approved by the Board of Directors of the American College of Medical Genetics

October 26, 2004

DOI: 10.1097/01.gim.0000177416.12323.58 ated with specific subtypes of malignancies that may be crucial for differential diagnosis. ${ }^{1,2}$

E6 (e) Monitoring the percentage of cases for which an abnormal clone has been identified is recommended for quality assurance assessment for the laboratory. Such monitoring is particularly valuable for cases of documented acute leukemia, in which the expected percentage with abnormalities is $>50 \%$; a lower than expected rate can alert the laboratory to issues such as tissue processing or analysis guidelines.

E6 (f) For studies performed, the cytogenetics laboratory should obtain, at the time of receipt of the specimen, as much information as possible about the suspected pathologic diagnosis, preliminary morphologic, immunophenotypic and clinical findings, results of any previous cytogenetic testing and indication for the requested study.

\section{E6.1 BONE MARROW/BLOOD: SPECIMEN PROCUREMENT AND PROCESSING}

Only those cells involved in the disease process will harbor the abnormalities being sought. The culture techniques and methodologies utilized should optimize the probability of detecting an abnormal clone.

E6.1.1 In most cases, bone marrow is the tissue of choice for analysis of suspected premalignant or malignant hematologic disorders.

In circumstances in which adequate bone marrow aspirate can not be obtained, alternative specimens may include:

E6.1.1 (a) Peripheral blood: (Blood specimens may yield informative results when the circulating blast count is higher than $10 \%$ to $20 \%$.) In general, the abnormal clone can be identified in such specimens, albeit not as often as in bone marrow. ${ }^{3}$ 
E6.1.1 (b) Bone marrow core biopsy (discussed under specimen collection, Section E6.1.2):

\section{E6.1.2 Specimen Collection}

E6.1.2 (a) Bone marrow and blood specimens should be collected under sterile conditions in sodium heparin. The concentration of sodium heparin should be approximately 20 units per $\mathrm{ml}$ of specimen (per either bone marrow volume alone, or per total volume of bone marrow and transport medium combined). If collected in a green top tube, the smaller sized sodium heparin tube should be used.

E6.1.2 (b) The volume of bone marrow available will differ for adults and children. An approximate specimen of 1 to $3 \mathrm{~mL}$ should be requested when possible. During the procurement of the specimen, several draws are likely to be taken from the patient. As the first draw is more concentrated with blasts, it is recommended that cytogenetics receive first or second draw whenever possible.

E6.1.2 (c) Specimens should be received by the laboratory as soon as possible (ideally within 24 hours).

E6.1.2 (d) It is generally recommended that specimens be maintained at ambient temperature during transit. Extreme temperatures should be avoided.

E6.1.2 (e) If a bone marrow core biopsy is obtained, it should be minced to generate cell suspensions. This can be done mechanically or enzymatically (e.g., with collagenase), if it is resistant to mechanical dissociation. Culture conditions are the same as those for bone marrow aspirates.

E6.1.2.2 Culture conditions should be optimized for the specific hematologic disorder suspected:

E6.1.2.2 (a) Acute leukemias: Unstimulated short-term cultures are recommended. If sufficient specimen is received, at least two cultures should be initiated, one of which should be designated as a 24-hour culture.

E6.1.2.2 (b) Aplastic anemia and chronic myeloproliferative disorders: Same as acute leukemias above.

E6.1.2.2 (c) Chronic lymphoproliferative disorders: Depending on the immunophenotype, additional cultures with B- or T-cell mitogens may be helpful.

E6.1.2.2 (d) Well-differentiated T-cell disorders (e.g., T-cell leukemia, T-cell lymphoma, Sezary syndrome, mycosis fungoides): T-cell mitogens may be helpful.

E6.1.2.2 (e) Mature B-cell disorders (e.g., plasma cell leukemia, multiple myeloma): Although there is no consensus on this point, some laboratories have had success in identifying abnormal clones with the addition of B-cell mitogens.

\section{E6.2 ANALYTICAL STANDARDS}

E6.2.1 Cell Selection. Cells selected for analysis should not be selected solely on the basis of good chromosome morphology. In general, the technologist should select an area of a slide to begin the analysis and then examine cells as they appear consecutively in the microscope field, only skipping cells for which extremely poor morphology precludes chromosome identification.

When cells are skipped because of poor morphology, it is important to attempt to count the number of chromosomes (particularly for possible hyper- or hypodiploidy relevant in pediatric ALL). In addition, attempts should be made to identify possible structurally abnormal chromosomes, particularly if the disease under consideration is associated with a specific recurring chromosome abnormality such as $\mathrm{t}(9 ; 22)$ in CML.

E6.2.2 Number of Cells Evaluated. The number of metaphase cells analyzed versus the number of metaphase cells counted or scored should be appropriate to the type of study (e.g., initial diagnosis vs. follow-up); the purpose of the study (e.g., detection of residual disease or response to therapy, monitoring for cytogenetic evolution or monitoring of engraftment of allogeneic transplant); and/or the characteristics of the specific chromosomal abnormalities present or suspected.

E6.2.2.1 Initial Diagnostic Studies

E6.2.2.1 (a) G-band analysis

Analysis: Analyze a minimum of 20 cells from unstimulated cultures. A combination of unstimulated and mitogen-stimulated cultures may be appropriate for the mature B- and T-cell disorders as described above (Section E6.1.2.2). For all other diagnoses (e.g., acute leukemias), only when the abnormal clone is identified in the mitogen-stimulated cultures can the G-band analysis include $<20$ metaphases from the unstimulated cultures.

\section{Documentation:}

- For the abnormal cells:

- If only one abnormal clone is present: Two karyotypes.

- If more than one related abnormal clone is present: Two karyotypes of the stemline and one of each sideline.

- If unrelated clones are present: Two karyotypes for each stemline and one for each associated pertinent sideline.

- For the normal cells:

- If only normal cells are present: Two karyotypes.

- If normal and abnormal cells are present: One karyotype of a normal cell.

E6.2.2.1 (b) Supplemental Florescent in situ hybridization (FISH) Analysis. Studies using FISH may be indicated to (1) provide a rapid result that will aid in the differential diagnosis or for planning induction therapy; or (2) to rule out a cryptic abnormality. Characterization of the initial diagnostic FISH pattern will also allow for future monitoring of the patient's disease. Examples of such cases include, but are not limited to:

(i) Acute promyelocytic leukemia (AML-M3): FISH with PML/RARA gene probes to detect $t(15 ; 17)$.

(ii) Chronic myelogenous leukemia: FISH with BCR/ABL gene probes for $\mathrm{t}(9 ; 22)$ or variants thereof.

(iii) Acute myelomonocytic leukemia with abnormal eosinophils (AML-M4eo): FISH with CBFB gene probe or other informative probe for inv(16) or $\mathrm{t}(16 ; 16)$.

(iv) Infant or childhood acute leukemia with high risk features: FISH with BCR/ABL and/or MLL gene probes.

(v) Precursor B-cell acute lymphoblastic leukemia most frequently seen in children ages 1 - 10 years: FISH with TEL/ AML1 gene probes to detect a cryptic 12;21 translocation. 
(vi) Suspected Burkitt leukemia/lymphoma (acute lymphoblastic leukemia L3): FISH with CMYC/IgH gene probes or others to detect $t(8 ; 14)$ or variant translocations.

(vii) B-cell CLL when a normal cytogenetic result is obtained by analysis of unstimulated and B-cell mitogen stimulated cultures to detect +12 , del(13)(q14), deletions of the ATM (11q22.3) and p53(17p13.1) genes.

Other applications of FISH include (but are not limited to), interphase FISH when there is insufficient material for G-banded analysis and there is a suspected pathologic diagnosis associated with a recurring chromosomal abnormality and; metaphase or interphase FISH to investigate the presence of a variant translocation suspected in the G-banding analysis by an abnormality involving a recurring chromosomal breakpoint.

Documentation: Documentation of FISH results should be in accord with Sections E9 and E10 of these Standards and Guidelines for Clinical Genetics Laboratories.

E6.2.2.2 Follow-up studies of patients who have had a previous cytogenetic study. For the following analytic guidelines, it is assumed that the laboratory has documentation of the patient's previous cytogenetic results. If the study has been performed elsewhere, and there is minimal information available, it is recommended that, except for patients seen for the first time post-transplant, the analysis be considered the same as an initial diagnostic workup (see above).

E6.2.2.2 (a) Patients who have NOT received allogeneic hematopoietic cell transplant (e.g., history of previous radiation and/or chemotherapy, autologous transplant, no prior therapy, etc.):

Analysis: Analyze 20 cells. If all cells are normal, additional cells may be scored for a specific abnormality by G-banding or FISH.

${ }^{\star}$ Note: For some patients, follow-up cytogenetic study is ordered to rule out a therapy-associated malignancy (e.g., MDS) rather than disease recurrence.

\section{Documentation:}

- For cases with both normal and abnormal cells or only abnormal cells:

- One karyotype of a normal cell, if such a karyotype was not documented in a previous study by the laboratory; otherwise, one normal metaphase spread.

- One or two karyotypes from each abnormal clone for a minimum total of two karyotypes.

- For cases with all normal cells:

- Two karyotypes.

E6.2.2.2 (b) Patients who are postallogeneic hematopoietic cell transplant for whom donor versus recipient origin of cells can be determined (by sex chromosome complement or cytogenetic heteromorphisms):

For studies aimed solely at determining engraftment status, molecular methods and/or interphase FISH (in the case of opposite sex transplant) are more sensitive than G-banded analysis and are the preferred methodologies. Therefore, in consultation with the referring physician, cancellation of test requests for G-band analysis for engraftment status should be considered.

During the course of the cytogenetic analysis it will become evident whether there is chimerism for donor and recipient cells. It is expected that there will be different approaches used by different laboratories to address these studies.

- If only donor cells are present:

Analysis: Analyze 20 cells.

Documentation: Document two karyotypes for each cell line. (In such cases, one is documenting either the constitutional karyotype (normal or abnormal of the donor) or the rare event of a malignant process arising in a donor cell.)

- If donor and recipient cells are present:

Analysis: Analyze recipient cells completely for previously identified clonal chromosome abnormalities and any newly acquired abnormalities. In some cases there may be structural chromosomal abnormalities secondary to chromosome breakage or rearrangement induced by the pretransplant conditioning regimen. The laboratory should distinguish clonal from nonclonal changes and determine the significance of new abnormalities as possible.

Analyze all recipient cells present out of 20 cells. Evaluate each recipient cell for the presence of the abnormality present prior to transplant (i.e., the diagnostic abnormality). Depending on the number of recipient cells present among the initial 20 metaphase cells scored, additional recipient cells may be analyzed completely and/or scored for the presence of the diagnostic abnormality.

Donor cells: Analyze two donor cells if donor cells have not been analyzed in previous studies. Otherwise simply score these cells as being of donor origin, and count.

Documentation: For the recipient cells: Two karyotypes of the stem line and one of each sideline.

For the donor cells: If donor cells have been documented previously, then provide a single metaphase spread. If donor cells have not been documented previously, provide two karyotypes.

- If only recipient cells are present:

Analysis: Analyze 20 cells, following the guidelines set forth above with respect to the characterization of secondary abnormalities.

Documentation: Same as above for abnormal recipient cells.

E6.2.2.2 (c) Patients posthematopoietic transplant for whom donor and recipient cells cannot be differentiated by G-banding:

Analysis: Analyze 20 cells. As in case scenarios outlined above, follow guidelines for recipient cells as set forth above.

\section{E6.3 TURNAROUND TIME (TAT) SHOULD BE APPROPRIATE FOR CLINICAL UTILITY.}

It should generally be recognized that under certain clinical circumstances, specific chromosome abnormalities, or lack thereof, may be crucial in establishing a diagnosis and have direct relevance to specific treatment offered to the patient. Every effort should be made to expedite conveying such chro- 
mosome information. It is strongly recommended that the cytogenetics laboratory director have a written policy describing how cases are prioritized in the laboratory, with associated TATs, and addressing the particular specimen processing and analytical needs.

E6.3.1 For initial diagnostic work-up, to rule out, confirm, or further characterize a diagnosis of acute lymphoblastic leukemia, acute myeloid leukemia, or chronic myelogenous leukemia, it is strongly recommended that these studies have preliminary results reported within 7 calendar days. Final results should be reported out within 21 calendar days.

E6.3.2 In the initial diagnostic work-up of other hematologic disorders, and for longitudinal studies on a patient, it is strongly recommended that final results be reported within 21 calendar days.

E6.3.3 Preliminary verbal reports and dates such results were given should be clearly documented in the final report. The content of the preliminary report should also be documented if it differs from that of the final report.

E6.3.4 Specific exceptions to these guidelines are: Diagnostic studies for acute promyelocytic leukemia should be approached on a STAT basis; as selection of induction chemotherapy (including or excluding the use of ATRA as part of the regimen) depends largely upon cytogenetic or molecular findings. Therefore, if APL is suspected in a patient, it is highly desirable for preliminary results of FISH and/or Gbanding to be reported within 2 working days of receipt of the specimen.

\section{E6.4 LYMPH NODES}

E6.4.1 Specimen Procurement and Processing. Common diagnoses include Hodgkin and non-Hodgkin lymphomas, including follicular, diffuse large B-cell, marginal zone, mantle cell, T-cell, and anaplastic large cell lymphoma.

E6.4.1 (a) Information regarding the pathologic examination of the tissue (e.g., whether or not a malignant process was identified, any subtyping that is available, including immunophenotype) can provide valuable information regarding the processing and analytical procedures to be followed.

E6.4.1 (b) Lymphoma may initially present as a mediastinal or other mass. Therefore, pathologic information should be pursued at the time of receipt of the tissue in the laboratory. If lymphoma is in the differential diagnosis, the cultures initiated should include at least one nonstimulated suspension culture as follows:

(i) In general, short-term (to include a 24-hour unstimulated culture) are preferred, with the number of cultures established being consistent with the apparent cellularity of the tissue specimen.

(ii) Of note, in suspected lymphoma cases, bone marrow aspirate is frequently sent for staging purposes, but if it shows no or limited involvement by lymphoma, conventional cytogenetics will most often yield normal chromosome findings. Therefore the laboratory should be sure to discuss with the clinicians the need to get a biopsy specimen from the involved lymph node or mass.

\section{E6.4.2 Analytical Standards}

E6.4.2 (a) Selection of cells to analyze: As with bone marrow and blood specimens, cells selected for analysis should not be chosen based on good chromosome morphology. After an area of a slide is chosen to begin analysis, the technologist should examine cells as they appear consecutively in the microscope field.

E6.4.2 (b)

Analysis: Analyze 10 to $20 \mathrm{G}$-banded metaphase cells. (Fewer than 20 metaphases is acceptable as long as the abnormal clone has been identified and characterized.)

${ }^{*}$ Note: Some lymphomas have highly complex karyotypes. In such cases, it may not be practical to analyze 20 metaphases. Therefore, in such studies, it is recommended that a sufficient number of metaphases (generally at least 10) be analyzed to permit characterization of the abnormal clone(s). The laboratory director should ensure that in the case of performing an abbreviated study, the CPT codes billed are consistent with the work performed as described in Section E3.2 of the Standards and Guidelines for Clinical Genetics Laboratories.

Rationale for the "Note": Unlike a study for aplastic anemia or myelodyplastic syndrome wherein the proportion of abnormal versus normal metaphase cells may have clinical significance, in the analysis of a lymph node or solid tumor, the sole clinical importance is the presence or absence of an abnormal clone, and the characterization of the chromosomal abnormalities involved. In a tissue in which the laboratory finds only abnormal cells, all with highly complex karyotypes, the goal should be to analyze a sufficient number to permit characterization of the clone. Extending the study to 20 cells could be very time- and resource-consuming, without any real added value to the diagnosis of the patient.

Documentation: Follow guidelines for initial diagnostic workup of blood and bone marrow (Section E6.2).

If there is confirmed pathologic diagnosis of lymphoma, and analysis of 20 metaphases yields only normal cells, additional screening for specific lymphoma-associated chromosomal rearrangements (e.g., the $2 ; 5,8 ; 14,11 ; 14$, or $14 ; 18$ translocations) can be performed by G-banding or FISH.

E6.4.3 Turnaround Time

E6.4.3 (a) It is recommended that $90 \%$ of specimens have final reports completed within 21 calendar days.

E6.4.3 (b) Certain patient cases may need preliminary and/or STAT verbal or written reports. The laboratory should obtain sufficient information at the time of specimen receipt to permit prioritization in the laboratory in accord with clinical need.

\section{E6.5 SOLID TUMORS}

It is critical that upon receipt in the laboratory of solid tumor material, as much information as possible be obtained regarding clinical information about the patient and suspected pathologic diagnosis.

\section{E6.5.1 Specimen Collection and Processing}

E6.5.1 (a) Specimens should be transported to the laboratory in sterile medium. If sterile medium is not available, sterile 
saline may be used. Optimally, $1 \mathrm{~cm}^{3}$ of specimen should be submitted for cytogenetic analysis.

E6.5.1 (b) Upon its receipt in the laboratory, the specimen should be examined under sterile conditions, and any obvious fat or necrotic tissue should be removed. The tissue should be minced, or enzymatically digested if tissue is resistant to mechanical dissociation.

E6.5.1 (c) To the extent possible, culture processing should be tailored to the suspected pathologic diagnosis.

Among solid tumors there is great diversity in the culture conditions that are optimal for growth of the malignant clone. For some, the malignant tumor will grow in suspension cultures; others will grow best in flask or coverslip cultures. For example:

(i) The small round blue cells tumors of childhood (Ewing/ PNET, neuroblastoma, Wilms) present a group of solid tumor studies for which cytogenetic findings can be critical for differential diagnosis. Either unstimulated suspension cultures or monolayer (coverslip) cultures have been reported to yield successful results.

(ii) For sarcomas and germ cell tumors, monolayer or coverslip cultures are likely to be most successful.

Some of these solid tissue specimens may represent lymphomas, and should be processed accordingly (Section E6.4). Others may represent extramedullary hematopoietic malignancies, and should be processed according to the bone marrow procedures (Section E6.3). This emphasizes the importance of obtaining as much information as possible regarding the differential diagnosis at the time of specimen receipt.

E6.5.1 (d) It is generally recommended that the monolayer or coverslip cultures initiated be harvested as soon as sufficient mitotic activity is present. There is some risk of overgrowth of normal tissue elements in long-term tissue culture.

E6.5.2 Analytical Methods. Analyze 10 to 20 metaphase cells by G-banding as described above for lymphomas. If the karyotype of the tumor is complex, 10 metaphases are likely to be sufficient to characterize the abnormal clone.

Documentation: Follow guidelines for initial diagnostic workup of blood and bone marrow (Section E6.2).

E6.5.3 Turnaround Time. It is recommended that $90 \%$ of cases have final reports out within 21 calendar days. The laboratory director should be apprised of special clinical issues that may require a shorter TAT for impacting patient care.

\section{References}

1. Jaffee ES, Harris NL, Stein H, Vardiman JW editors. World Health Organization classification of tumours: pathology and genetics of tumours of hematopoietic and lymphoid tissues, vol. 3. Lyon: IARC Press, 2001.

2. Mitelman F, Johansson B and Mertens F (editors).Mitelman Database of Chromosome Aberrations in Cancer (2004).$<$ http://cgap.nci.nih.gov/Chromosomes/Mitelman $>$

3. Weinkauff R, Estey EH, Starostik P, Hayes K, Huh YO, Hirsch-Ginsberg C, et al. Use of peripheral blood blasts versus bone marrow blasts for diagnosis of acute leukemia. Am J Clin Pathol 1999;111:733-740. 